\title{
PROPUESTA METODOLÓGICA PARA LA CONSTRUCCIÓN DE INDICADORES SINTÉTICOS DE IGUALDAD DE GÉNERO El caso del medio rural de Castilla y León
}

\author{
A METHODOLOGICAL PROPOSAL TO BUILD COMPOSITE INDICATORS \\ OF GENDER EQUALITY \\ The Case of Rural Areas in Castilla y León
}

\author{
MARGARITA RICO \\ Universidad de Valladolid \\ mrico@iaf.uva.es
}

José ANTONIO GÓMEZ-LIMÓN

Instituto de Investigación y Formación Agraria y Pesquera (IFAPA). Córdoba

josea.gomezlimon@juntadeandalucia.es

\begin{abstract}
RESUMEN
La desigualdad socioeconómica por razón de género constituye un problema multidimensional difícil de cuantificar en su globalidad. La utilización de indicadores sintéticos puede ayudar a resumir e interpretar el conjunto de información de que se disponga al respecto, así como evaluar las disparidades en la situación de mujeres y hombres desde un punto de vista estático y también dinámico. Dicha utilidad se magnifica para el estudio de las diferencias por sexo en los espacios rurales, dadas las especiales carencias con las que se enfrentan las mujeres que allí residen. Por todo ello, el objetivo de este trabajo consiste en desarrollar una metodología para construir indicadores sintéticos de igualdad de género que cuantifiquen de forma agregada la existencia de asimetría de género en territorios heterogéneos. Con el fin de validar esta propuesta metodológica, se ha realizado una aplicación empírica de los indicadores sintéticos planteados para la Comunidad Autónoma de Castilla y León, comparando el desempeño de estos índices en distintos tipos de hábitats: rural, intermedio y urbano.
\end{abstract}

\section{Palabras Clave}

Análisis cuantitativo, Brecha de género, Castilla y León, Mujer rural.

\footnotetext{
ABSTRACT

The socioeconomic unequality between genders is a multidimensional problem difficult to be quantified as a whole. The use of composite indicators can help in this task by summarizing and interpreting the complete set of available information. Furthermore, these indexes can be used to analysis the disparities in the situation of women and men from a static point of view and also from a dynamic perspective. The usefulness of these composite indicators is even more important for the study of gender gap in rural areas, given the particular difficulties faced by women living in these districts. In this sense, the objective of this paper is to develop a methodology to build composite indicators of gender equity that quantifies the existence of asymmetry between
} 
men and women in different kind of habitats. In order to validate the methodological approach proposed, this has been implemented for the Autonomous Region of Castilla y León (Spain). This case study is focused in the calculation of composite indicators that allow the comparison of gender gaps in rural, intermediate and urban areas.

\section{KEYWORDS}

Castilla y León (Spain), Gender Gap, Quantitative Analysis, Rural Women.

\section{INTRODUCCIÓN $^{1}$}

Hasta la década de los 70 las organizaciones responsables del desarrollo no consideraban las desigualdades existentes por razón de género como un tema relevante. El papel asignado a la mujer se limitaba a las facetas reproductiva y doméstica. En este sentido, el trabajo de Boserup (1970) fue el primero que puso de manifiesto que el crecimiento económico que se estaba produciendo en esos momentos no sólo no beneficiaba a las mujeres, sino que las perjudicaba, reconsiderándose así las distintas políticas de desarrollo que se estaban aplicando. Desde entonces, se comenzó a incorporar a las mujeres en las corrientes de desarrollo (Calatrava 2002), aunque aún persisten importantes discriminaciones sobre todo en países menos avanzados. En la Unión Europea el principio de igualdad de oportunidades se considera actualmente una prioridad transversal dentro del ordenamiento jurídico comunitario y los esfuerzos por conseguir la igualdad se han de materializar en el conjunto de acciones y políticas generales implementadas (Rico 2006).

En el caso de las mujeres que habitan las zonas rurales, las características diferenciales en su situación socioeconómica con respecto a los hombres o con respecto a las mujeres de zonas urbanas (falta de acceso a los servicios, falta de oferta de empleo apropiado, etc.) han hecho imprescindible la instrumentalización de medidas públicas que se ajusten a sus necesidades. Estas acciones constituyen un importante apoyo, debido al generalizado proceso de incorporación al mercado de trabajo en el medio rural que están protagonizando, contribuyendo así al dinamismo de estas zonas (García Ramón y Baylina 2000; García Sanz 2004).

Si bien son numerosos los trabajos publicados desde los años 70 acerca de la situación socioeconómica de las mujeres en la agricultura y en el medio rural, es preciso destacar los de García Ferrando (1977), Cánoves et al. (1989), Sabaté (1989), García Bartolomé (1990), Vicente Mazariegos (1993), Sampedro (1996), Instituto de la Mujer (1999), García Ramón y Baylina (2000), García Sanz (2004) o Camarero (2006).

\footnotetext{
${ }^{1}$ Los autores agradecen sinceramente las aportaciones realizadas por los revisores anónimos, en la medida que han permitido una mejora sustancial del documento original. Esta investigación ha sido financiada por el Ministerio de Educación y Ciencia (MEC-FEDER) a través del proyecto FUTURPAC (AGL200605587-C04-01).
} 
Tanto la literatura al respecto como las diferentes organizaciones nacionales y supranacionales insisten en la necesidad de que se elaboren estadísticas e indicadores que muestren la situación específica de las mujeres que viven en las zonas rurales y evalúen la eficacia de las medidas y los programas de acción que se están aplicando en cada zona (Organización de las Naciones Unidas 1984; Food and Agriculture Organization of the United Nations 2001; Comisión Económica para América Latina y el Caribe 2006). En el caso español, la Carta para la lgualdad de Oportunidades de las Mujeres en el Ámbito Rural (Instituto de la Mujer 1995), recoge la necesidad de reflejar en las estadísticas oficiales los trabajos que realizan las mujeres rurales y conseguir el reconocimiento de sus aportaciones a la economía rural. Por su parte, el Plan de igualdad entre mujeres y hombres en el medio rural (Ministerio de Agricultura, Pesca y Alimentación 2007) señala entre sus actuaciones la necesidad de mejorar sus estadísticas, desagregando los datos según la variable sexo y propone realizar estudios para profundizar en el conocimiento de la situación de este colectivo.

Al hilo de lo expuesto anteriormente, se necesita de una estructura estadística sistematizada que recoja información sobre la situación de las mujeres y también de los varones en las distintas esferas económicas y sociales de la vida, habida cuenta de que la desigualdad de género es un problema multidimensional que afecta al ámbito demográfico, económico, político, social, educativo, etc. Así, surgen los "sistemas de indicadores de género" como una herramienta útil para identificar los cambios en el estatus y en el rol de las mujeres a lo largo del tiempo, así como para evaluar si la igualdad de oportunidades está siendo alcanzada por medio de las acciones implementadas a tal fin (Álvaro 1994; Dávila 2004).

La cuantificación de la igualdad de género a través de indicadores, sin embargo, plantea ciertos problemas, relacionados con la complejidad para interpretar conjuntamente la multiplicidad de variables que requiere este tipo de análisis, circunstancia que obstaculiza su utilización como herramienta práctica de apoyo a la toma de decisiones públicas. Este problema ha tratado de superarse a través de la aplicación de métodos de agregación de estos conjuntos multidimensionales de indicadores en "índices" o "indicadores sintéticos"2, que resuman esta amplia información. En este sentido, es escasa la literatura que aborda la sistematización de un indicador sintético para reflejar las diferencias por razón de sexo en cualquier ámbito socioeconómico, destacando a nivel internacional el Informe sobre Desarrollo que anualmente publica el Programa de Naciones Unidas para el Desarrollo (PNUD 2007), en el que se calcula el Índice de Desarrollo Humano relativo al Género (IDG) y el Índice de Potenciación de Género (IPG). Existen otros cuatro indicadores sintéticos elaborados por otros tantos organismos internacionales, que se utilizan para medir la inequidad de género: el Índice de Disparidad entre Géne-

\footnotetext{
2Debe indicarse que los términos "índice" (index en inglés) e "indicador sintético" o "compuesto" (composite indicator) deben considerarse sinónimos. En el documento se ha optado por emplear preferentemente el segundo de ellos, al objeto de denotar el carácter multiatributo de los índices construidos.
} 
ros (World Economic Forum 2008); el Índice de Equidad de Género (Social Watch)2009) el Índice de Género e Instituciones Sociales de la Organización para la Cooperación y el Desarrollo Económico -OCDE- (Jütting et al. 2006) y el Índice de Desarrollo de Género para África (United Nations Economic Commision For Africa 2004). Todos ellos se construyen a un nivel territorial macroagregado (a nivel de país) y para su elaboración se toman en consideración un número reducido de variables (desde las 4 variables del IDG y el IPG hasta las 14 que componen el Índice de Disparidad entre Géneros).

En cuanto a investigaciones específicas acerca del tema, cabe citar el trabajo de Castañeda et al. (1999), donde se construye un indicador sintético para medir diferencias socioeconómicas de género entre diversos territorios de Cuba. Para el caso español, el Barómetro Social de España (Colectivo loé 2008) elabora un Índice Sintético de lgualdad de Género para el conjunto nacional que consta de cuatro dimensiones (actividad laboral, salud, formación y protección social) y 27 variables. Por su parte, Bericat y Sánchez (2008) proponen un Índice de Desigualdad de Género a partir de 23 indicadores referidos a tres ámbitos públicos: educativo, laboral y de poder. No obstante, no se tiene constancia de trabajos donde se calculen indicadores sintéticos de igualdad de género y se realicen comparaciones entre distintos tipos de hábitat.

En consecuencia, y dada la exigua investigación al respecto, el presente trabajo tiene como principal objetivo desarrollar una metodología para la construcción de indicadores sintéticos de igualdad de género que cuantifiquen, de forma agregada y resumida, la existencia de asimetría de género en territorios heterogéneos. Esta propuesta metodológica pretende tener una validez general para determinar las diferencias entre hombres y mujeres en cualquier tipo de hábitat (rural o urbano).

Además, al objeto de validar la propuesta metodológica, este trabajo pretende realizar una implementación de los índices planteados para las zonas rurales de la Comunidad Autónoma de Castilla y León, comparando el desempeño de los índices propuestos en dichos territorios con los correspondientes valores de las zonas urbanas de dicha región. El interés objetivo de esta aplicación empírica reside en la difícil situación a la que se enfrentan las mujeres en dichas zonas, así como las importantes carencias de información sensible al género. Los resultados tratarán de cubrir las carencias de conocimiento antes aludidas, evidenciando que su empleo puede representar un instrumento potencialmente útil para los decisores políticos a la hora de elaborar, implementar y evaluar estrategias tendentes a la consecución de la igualdad de oportunidades en este tipo de hábitat.

Para alcanzar los objetivos antes dispuestos, el trabajo se ha estructurado como sigue: tras esta sección introductoria, en el segundo apartado se justifica el ámbito territorial utilizado como caso de estudio, esto es, la Comunidad Autónoma de Castilla y León y, en particular, su territorio rural. El tercer apartado detalla la metodología empleada para la construcción de los indicadores sintéticos. En la cuarta sección se detallan los principales resultados obtenidos en la aplicación empírica. Para finalizar, el quinto apartado recoge las principales conclusiones del trabajo. 


\section{El Caso De Estudio}

Castilla y León es, probablemente, la región española que más está padeciendo los efectos de la crisis rural, materializada en la acentuada despoblación de sus pequeños municipios, el continuado envejecimiento y la acuciante masculinización y, en definitiva, la descapitalización demográfica y económica de buena parte de su territorio (Rico y Gómez-Limón 2008).

El proceso de modernización y tecnificación agrario de los años 60 y 70 se produjo sin apenas protagonismo perceptible de las mujeres, aunque ellas siempre aportaron su inestimable trabajo para sacar adelante la explotación familiar (García Bartolomé 1990). Sin embargo, este trabajo de las mujeres rurales no ha sido reconocido social ni económicamente (Vera y Rivera 1999), una de las causas que las empujaron a abandonar el campo 0 a que sus hijas lo hicieran, en busca de un empleo visible que les diera independencia económica y social (De la Fuente 1987; Sampedro 1996). Las consecuencias se reflejan en unas zonas rurales envejecidas y masculinizadas, factores éstos limitantes para la reestructuración del tejido económico rural y de la sociedad. Así, las principales características socioeconómicas que definen al colectivo de mujeres rurales en Castilla y León se sintetizan en los siguientes aspectos:

- Desde mediados del siglo XX se ha producido un progresivo deterioro demográfico en el medio rural castellano y leonés, sobre todo por parte del colectivo femenino. El movimiento migratorio selectivo ha ocasionado que hayan sido sobre todo las mujeres más jóvenes y cualificadas las que han optado por abandonar los pequeños municipios. Adicionalmente, las mujeres tienen una mayor reticencia que los varones a fijar su residencia en un territorio rural, con lo que sus saldos migratorios son más negativos que los de los varones (Sampedro 1996; Camarero y Sampedro 2008).

- Este proceso de despoblamiento rural determina la evolución de otras variables demográficas tales como la natalidad, la nupcialidad y la fecundidad. En un territorio con ausencia de población joven femenina parece lógico que se reduzca el número de nacimientos, de matrimonios y de hijos por mujer. Todos estos hechos provocan una retroalimentación del círculo vicioso de la despoblación, y que estas zonas cada vez sufran en mayor medida la ausencia de habitantes y sus consecuencias, tanto demográficas como laborales y sociales (Instituto de la Mujer 1999). Hay que señalar asimismo el alto índice de envejecimiento rural que afecta en mayor proporción a las mujeres debido a su mayor esperanza de vida.

- La proporción de población inactiva de las mujeres del ámbito rural de Castilla y León es más elevada que en otras zonas más pobladas. Aunque en los últimos años la cantidad de activas ha aumentado, el camino por recorrer hasta equipararse a las tasas masculinas aún es largo (Rico 2006). Asimismo, destaca la mayor tasa de paro de las mujeres rurales con respecto a los varones, 
su menor retribución y su escasa participación en puestos de dirección y control (Consejo Económico y Social de Castilla y León 2004). Además, la división del trabajo existente sigue reproduciendo los roles tradicionales femeninos, y la mayor carga de la mujer en cuanto al trabajo reproductivo condiciona la incorporación femenina a puestos de trabajo indefinidos y a tiempo completo.

- Si bien las mujeres rurales han tenido tradicionalmente un nivel educativo más bajo que las de las ciudades, por las menores posibilidades para desarrollar una actividad educativa dentro de un pueblo, es importante señalar que en estos espacios el nivel de estudios femenino supera al masculino (Sampedro 1996), sobre todo entre los más jóvenes, como así lo testimonia por ejemplo la mayor proporción de mujeres universitarias en relación a los varones. Este hecho es indicativo del esfuerzo considerable que está realizando este colectivo para mejorar su formación y equiparar así sus posibilidades de empleo (Díaz Méndez 2006; Cruz 2006). En cuanto a la participación política femenina, si bien cada vez más mujeres están ocupando puestos de representación a escala local, regional o nacional, esta participación aún es muy reducida al compararla con los hombres (Consejo Económico y Social de Castilla y León 2004).

A tenor de lo descrito, la implementación de medidas públicas para corregir las desigualdades existentes demandan la existencia de estudios e indicadores estadísticos específicos que describan: (a) la actual situación de estas mujeres en todos los ámbitos de su vida, (b) la heterogeneidad del propio colectivo dependiendo de características como su edad, su formación o su nivel de renta, (c) la posición relativa con respecto a los varones y a las mujeres de áreas más pobladas, y (d) la evolución de estas disparidades a lo largo del tiempo.

\section{Metodología}

\section{El sistema de indicadores para el análisis cuantitativo de la igualdad de género}

Como paso previo a la generación de un indicador sintético, es preciso contar con una serie de indicadores simples suficientemente explicativos de la realidad que se pretenda mensurar. A este respecto, se puede definir un "indicador de género" como aquél cuya finalidad reside en recoger los cambios sociales debidos a las relaciones entre mujeres y hombres a lo largo del tiempo (Dávila 2004). Consecuentemente, para analizar un fenómeno multidimensional, como lo es la igualdad entre hombres y mujeres, se hace necesaria información sobre un amplio conjunto de variables, todas ellas recogidas en un sistema de indicadores de género. Este sistema de indicadores debe proporcionar información sobre la situación de las mujeres en múltiples ámbitos, así como las posibles desigualdades existentes al compararlas con el colectivo masculino y con mujeres pertenecientes a otros territorios. 
Lo primero que se ha tenido en cuenta, antes de comenzar con la selección de indicadores propiamente dicha, es el contexto o situación actual existente y la situación deseable u objetivos que se pretenden alcanzar en el futuro (Agencia Canadiense de Desarrollo Internacional 1996; Dávila 2004; Comisión Económica para América Latina y el Caribe, 2006). Así, a través de una amplia revisión bibliográfica, se han determinando las principales características del colectivo de mujeres que viven en el ámbito rural de Castilla y León, así como los principales objetivos que persiguen las políticas de igualdad de oportunidades en los territorios rurales, ligando así los objetivos planteados desde las estrategias puestas en marcha en un determinado momento con los indicadores propuestos en el sistema.

En ocasiones, los objetivos políticos perseguidos pueden ser claros y fácilmente cuantificables, pero, en general, en el caso de la consecución de la igualdad de oportunidades la identificación de objetivos precisos es más complicada. Se trata de procesos sociales de cambio, donde los objetivos no se concretan de forma numérica, sino que se fijan hacia la reducción de desigualdades entre hombres y mujeres en distintos aspectos, tal y como señalan las instituciones más relevantes al respecto (Organización de las Naciones Unidas 1995; Food and Agriculture Organization of the United Nations 2001; Comisión Europea 2006; Ministerio de Igualdad 2007; Instituto de la Mujer 1995; Ministerio de Agricultura, Pesca y Alimentación 2007; Junta de Castilla y León 2007), como las organizaciones sociales (asociaciones de mujeres y sindicatos) y los estudios e investigaciones realizados (Álvaro 1994; Dávila 2004). Por consiguiente, y teniendo en cuenta los ámbitos socioeconómicos más significativos en los que existen disparidades intragénero (Álvaro 1994) y dependiendo de los objetivos de política pública que se pretendan alcanzar, los indicadores de género considerados se han distribuido en siete áreas temáticas: (a) Población y Familia; (b) Educación y Formación; (c) Empleo y Renta; (d) Participación Política y Social; (e) Usos del Tiempo; (f) Calidad de Vida; (g) Opiniones sobre la Igualdad de Oportunidades.

Complementariamente, para que un indicador de género cumpla el objetivo para el cual se ha diseñado, requiere que recoja la mayor cantidad posible de información relativa a la realidad a analizar (Dávila 2004). En consecuencia, la selección de indicadores de género se ha efectuado teniendo en cuenta ciertas características básicas que todo indicador ha de cumplir (Álvaro 1994): relevancia, validez, fiabilidad, perdurabilidad, comprensibilidad, sensibilidad y viabilidad. Teniendo en cuenta tales criterios, la selección de indicadores vinculados a cada uno de los objetivos se ha realizado, en primera instancia, a partir de una extensa revisión bibliográfica. Posteriormente, la propuesta desarrollada por los autores ha sido debatida y consensuada con un panel de 11 expertos en materia de igualdad de géneros que han participado en este estudio. Dicho panel ha estado compuesto por personas de reconocido prestigio extraídas del ámbito universitario (5), de las administraciones públicas (4) y de asociaciones de mujeres (2). Ocho de ellas eran mujeres y tres eran varones.

Con este procedimiento se ha tratado de minimizar la subjetividad y la arbitrariedad inherentes a todo proceso de selección de indicadores, tratando de ajustar tal selección 
al estado actual de conocimiento en relación al tema (revisión de literatura y consulta de expertos). En todo caso, siguiendo la filosofía del conjunto de la investigación, debe entenderse la selección finalmente realizada como una propuesta, que junto con otras aportaciones similares debe ser objeto de revisión hasta alcanzar el necesario consenso para su implementación operativa a gran escala.

Adicionalmente, los indicadores seleccionados, obviamente, deben estar desagregados por sexo. Dada la finalidad de este trabajo, además, estos han de estar desglosados según distintos ámbitos territoriales, con el fin de establecer comparaciones entre diversos hábitats. Con este propósito se ha seguido un criterio de ruralidad que distingue entre zona "rural" (municipios de menos de 2.000 habitantes) 33; zona "semirrural" o "intermedia" (municipios de entre 2.000 y 10.000 habitantes) y zona "urbana" (municipios de más de 10.000 habitantes). Finalmente, los indicadores seleccionados se han desagregado igualmente para 3 cohortes distintas de edad: población "joven" (entre 16 y 29 años); población "madura" (entre 30 y 64 años); y población "mayor" (más de 64 años).

En la tabla 1 se ofrece el listado de indicadores de género finalmente seleccionado, atendiendo a los criterios descritos y relacionados con los objetivos de política económica que se desean alcanzar. Se trata de un total de 26 indicadores simples, repartidos en las 7 áreas temáticas anteriormente aludidas y cuya descripción básica, fuente y año también se incluyen en la citada tabla.

\footnotetext{
${ }^{3}$ El Instituto Nacional de Estadística (INE) utiliza ese mismo criterio de ruralidad, si bien referido a entidades singulares de población en vez de a municipios. No obstante, dada la exigua información existente con desagregación a nivel de entidad singular, la mayor parte de los trabajos de investigación utilizan el municipio como unidad de medida a la hora de determinar el grado de ruralidad. A este respecto puede consultarse, por ejemplo, García Sanz (1998) o Sancho y Reinoso (2003).
} 
Tabla 1.

Descripción de los indicadores sintéticos de base (I).

\begin{tabular}{|c|c|c|c|}
\hline OBJETIVO & INDICADOR & DESCRIPCIÓN & FUENTE Y AÑO \\
\hline \multicolumn{4}{|c|}{ A) POBLACIÓN Y FAMILIA } \\
\hline $\begin{array}{l}\text { Fijar población en el } \\
\text { territorio }\end{array}$ & Indicador poblacional & $\begin{array}{l}\text { Proporción de mujeres } \\
\text { y varones entre la } \\
\text { población total }\end{array}$ & Padrón INE. 2008 \\
\hline $\begin{array}{l}\text { Detener el progresivo } \\
\text { envejecimiento poblacional }\end{array}$ & Indicador de juventud & $\begin{array}{l}\text { Proporción de mujeres } \\
\text { y varones entre } 15 \text { y } \\
64 \text { años }\end{array}$ & Padrón INE. 2008 \\
\hline $\begin{array}{l}\text { Frenar el éxodo } \\
\text { demográfico }\end{array}$ & Saldo migratorio & $\begin{array}{l}\text { Diferencia entre } \\
\text { inmigraciones y } \\
\text { emigraciones de } \\
\text { mujeres y varones }\end{array}$ & $\begin{array}{l}\text { Variaciones } \\
\text { Residenciales. INE. } \\
2002\end{array}$ \\
\hline $\begin{array}{l}\text { Detener el vaciamiento } \\
\text { demográfico }\end{array}$ & Saldo vegetativo & $\begin{array}{l}\text { Diferencia entre } \\
\text { nacimientos y } \\
\text { defunciones de mujeres } \\
\text { y varones }\end{array}$ & $\begin{array}{l}\text { Movimiento Natural } \\
\text { de la Pobl. INE. } 2006\end{array}$ \\
\hline \multicolumn{4}{|c|}{ B) EDUCACIÓN Y FORMACIÓN } \\
\hline Aumentar el nivel educativo & $\begin{array}{l}\text { Estudios } \\
\text { universitarios }\end{array}$ & $\begin{array}{l}\text { Mujeres y varones que } \\
\text { han cursado estudios } \\
\text { universitarios }\end{array}$ & $\begin{array}{l}\text { Encuesta ad hoc. } \\
2008\end{array}$ \\
\hline Facilitar la movilidad & Carné de conducir & $\begin{array}{l}\text { Mujeres y varones con } \\
\text { carné de conducir }\end{array}$ & $\begin{array}{l}\text { Encuesta ad hoc. } \\
2008\end{array}$ \\
\hline $\begin{array}{l}\text { Aumentar la formación } \\
\text { laboral }\end{array}$ & Cursos formativos & $\begin{array}{l}\text { Mujeres y varones que } \\
\text { han realizado cursos de } \\
\text { formación laboral }\end{array}$ & $\begin{array}{l}\text { Encuesta ad hoc. } \\
2008\end{array}$ \\
\hline Aumentar el uso de TICs & $\begin{array}{l}\text { Conocimientos de } \\
\text { informática }\end{array}$ & $\begin{array}{l}\text { Mujeres y varones que } \\
\text { tienen conocimientos de } \\
\text { informática }\end{array}$ & $\begin{array}{l}\text { Encuesta ad hoc. } \\
2008\end{array}$ \\
\hline
\end{tabular}

\section{C) EMPLEO Y RENTA}

Aumentar la incorporación

al mercado laboral

Disminuir el paro

Disminuir la precariedad

laboral

Aumentar la participación en los puestos de dirección

Eliminar la diferencia de rentas
Mujeres y varones activos

Mujeres y varones desempleados

Tiempo de duración del contrato

Ocupación por tipo de cualificación

Nivel de ingresos
Mujeres y varones con contrato indefinido

Mujeres y varones con puestos de dirección Mujeres y varones con ingresos mayores de 1.000 euros
Encuesta ad hoc.

2008

Encuesta ad hoc. 2008

Encuesta ad hoc. 2008

Encuesta ad hoc. 2008

Encuesta ad hoc. 2008 
Tabla 1 bis.

Descripción de los indicadores sintéticos de base (y II).

\begin{tabular}{|c|c|c|c|}
\hline OBJETIVO & INDICADOR & DESCRIPCIÓN & FUENTE Y AÑO \\
\hline \multicolumn{4}{|c|}{ D) PARTICIPACIÓN POLÍTICA Y SOCIAL } \\
\hline $\begin{array}{l}\text { Promover la participación en } \\
\text { las estructuras sociales }\end{array}$ & $\begin{array}{l}\text { Participación } \\
\text { asociativa }\end{array}$ & $\begin{array}{l}\text { Mujeres y varones } \\
\text { que pertenecen a una } \\
\text { asociación }\end{array}$ & $\begin{array}{l}\text { Encuesta ad hoc. } \\
2008\end{array}$ \\
\hline $\begin{array}{l}\text { Promover la participación } \\
\text { en las estructuras de poder } \\
\text { social }\end{array}$ & $\begin{array}{l}\text { Participación } \\
\text { asociativa por tipo de } \\
\text { contribución }\end{array}$ & $\begin{array}{l}\text { Mujeres y varones que } \\
\text { pertenecen a la directiva } \\
\text { de una asociación }\end{array}$ & $\begin{array}{l}\text { Encuesta ad hoc. } \\
2008\end{array}$ \\
\hline $\begin{array}{l}\text { Promover la participación en } \\
\text { la política }\end{array}$ & $\begin{array}{l}\text { Participación en } \\
\text { órganos políticos }\end{array}$ & $\begin{array}{l}\text { Mujeres y varones que } \\
\text { han ostentado un cargo } \\
\text { político municipal }\end{array}$ & $\begin{array}{l}\text { Encuesta ad hoc. } \\
2008\end{array}$ \\
\hline
\end{tabular}

\section{E) USOS DEL TIEMPO}

Aumentar la incorporación al trabajo remunerado

Avanzar hacia el reparto igualitario de las tareas del hogar

Compatibilizar el trabajo con la vida personal Dedicación al trabajo
remunerado

Dedicación al hogar

Dedicación al ocio
Porcentaje de tiempo dedicado al trabajo remunerado de mujeres y varones

Porcentaje de tiempo dedicado a las tareas del hogar de mujeres y varones

Porcentaje de tiempo dedicado al ocio de mujeres y varones
Encuesta ad hoc.

2008

Encuesta ad hoc.

2008

Encuesta ad hoc.

2008

\section{F) CALIDAD DE VIDAa}

\begin{tabular}{llll}
\hline $\begin{array}{l}\text { Mejorar el bienestar y la cali- } \\
\text { dad de vida en el ámbito } \\
\text { familiar }\end{array}$ & Vida familiar y social & $\begin{array}{l}\text { Satisfacción media de muje- } \\
\text { res y varones ante su vida } \\
\text { familiar y social }\end{array}$ & $\begin{array}{l}\text { Encuesta ad hoc. } \\
2008\end{array}$ \\
\hline $\begin{array}{l}\text { Mejorar el bienestar y la cali- } \\
\text { dad de vida en el ámbito } \\
\text { económico }\end{array}$ & Vida laboral y económica & $\begin{array}{l}\text { Satisfacción media de muje- } \\
\text { res y varones ante su vida } \\
\text { laboral y económica }\end{array}$ & $\begin{array}{l}\text { Encuesta ad hoc. } \\
2008\end{array}$ \\
\hline $\begin{array}{l}\text { Dotar de mayor número de } \\
\text { servicios sociales }\end{array}$ & $\begin{array}{l}\text { Acceso a los servicios } \\
\text { sociales }\end{array}$ & $\begin{array}{l}\text { Satisfacción media de muje- } \\
\text { res y varones ante el acceso } \\
\text { a los servicios sociales }\end{array}$ & Encuesta ad hoc. \\
2008
\end{tabular}




\section{G) OPINIONES SOBRE LA IGUALDAD DE OPORTUNIDADES ${ }^{b}$}

\begin{tabular}{llll}
\hline $\begin{array}{l}\text { Eliminar los estereotipos } \\
\begin{array}{l}\text { sexistas en el mercado } \\
\text { laboral }\end{array}\end{array}$ & $\begin{array}{l}\text { Responsabilidad en el } \\
\text { trabajo }\end{array}$ & $\begin{array}{l}\text { Opiniones de mujeres y } \\
\text { varones sobre la igualdad } \\
\text { en la responsabilidad } \\
\text { laboral }\end{array}$ & $\begin{array}{l}\text { Encuesta ad hoc. } \\
2008\end{array}$ \\
\hline $\begin{array}{l}\text { Eliminar los estereotipos } \\
\text { sexistas en el hogar }\end{array}$ & $\begin{array}{l}\text { Reparto de tareas del } \\
\text { hogar }\end{array}$ & $\begin{array}{l}\text { Opiniones de mujeres y } \\
\text { varones sobre el reparto de } \\
\text { tareas del hogar }\end{array}$ & Encuesta ad hoc. \\
\hline $\begin{array}{l}\text { Eliminar los estereotipos } \\
\text { sexistas en las estructuras } \\
\text { sociales y políticas }\end{array}$ & $\begin{array}{l}\text { Igualdad en el liderazgo } \\
\text { social y político }\end{array}$ & $\begin{array}{l}\text { Opiniones de mujeres y } \\
\text { varones sobre la igualdad } \\
\text { en el liderazgo social y } \\
\text { político }\end{array}$ & Encuesta ad hoc. \\
\hline
\end{tabular}

Fuente: Elaboración propia.

a Los indicadores del área "Calidad de vida" se han cuantificado a través de la media de las opiniones vertidas en la encuesta acerca de la satisfacción percibida por hombres y mujeres en diversas dimensiones de sus vidas, empleando para ello una escala Likert de 1 (mínima satisfacción) a 5 (máxima satisfacción).

" Los indicadores del área "Opinión sobre la igualdad de oportunidades" también se han cuantificado con una escala Likert de 1 a 5 según las opiniones de los individuos encuestados acerca de la necesidad o no de avanzar en materia de igualdad en varios ámbitos de la esfera económica y social. 
La información necesaria para calcular los indicadores pertenecientes a los diversos ámbitos socioeconómicos proviene de dos tipos de fuentes: fuentes de información ya existentes y fuentes de información primaria, debido a la falta de información actualizada y desagregada por tipo de hábitat y sexo de muchas de las variables y a la simple inexistencia de otros datos, en especial los de carácter cualitativo. Consecuentemente, en el presente estudio se ha realizado una encuesta ad hoc, teniendo en cuenta todos aquellos datos que no eran ofrecidos por las fuentes estadísticas secundarias. Para ello, se ha elaborado un cuestionario específico ${ }^{4}$, distribuido en siete bloques (coincidiendo con las siete áreas temáticas anteriormente enumeradas) con 57 preguntas cerradas dirigidas a una muestra representativa del conjunto de la sociedad castellana y leonesa, compuesta por 707 hombres y mujeres mayores de 16 años y que residen en esta Comunidad Autónoma (Tabla 2).

Tabla 2.

Ficha técnica de la encuesta.

\begin{tabular}{ll}
\hline Población objeto de estudio & $\begin{array}{l}\text { Personas residentes en Castilla y León con edades iguales o } \\
\text { superiores a 16 años (2.557.330 personas según en Padrón de } \\
\text { Habitantes de 2008) }\end{array}$ \\
\hline Tamaño de la muestra & 707 entrevistas \\
\hline Tipo de entrevista & Encuesta telefónica, asistida por ordenador \\
\hline Tipo de muestreo & $\begin{array}{l}\text { Polietápico, estratificado por conglomerados (afijación proporcional } \\
\text { al tamaño del hábitat), con selección de las unidades primarias de } \\
\text { muestreo (municipios) mediante un procedimiento aleatorio propor- } \\
\text { cional, y selección de las unidades últimas de muestreo (individuos) } \\
\text { mediante rutas aleatorias y cuotas de sexo y edad }\end{array}$ \\
\hline Error & $\begin{array}{l}\text { El nivel de error absoluto máximo esperado de los resultados de } \\
\text { la encuesta, para las frecuencias de cada variable, es de } \pm 3,76 \%, \\
\text { para un nivel de confianza del } 95 \%\end{array}$ \\
\hline Fechas de trabajo de campo & Del 25 de agosto al 12 de septiembre de 2008 \\
\hline
\end{tabular}

Fuente: Elaboración propia.

\footnotetext{
${ }^{4}$ El cuestionario utilizado para la encuesta se encuentra a disposición de cualquier persona interesada mediante petición directa (por correspondencia) a la autora.
} 


\section{Metodología para la construcción de los indicadores sintéticos de igualdad de género}

Etapas para la construcción de un indicador sintético

Con objeto de dotar al proceso de construcción de Indicadores Sintéticos de Igualdad de Género (ISIG) de una secuencia lógica y coherente, que facilite su posterior comprensión y aplicabilidad, se han seguido las orientaciones propuestas por la OECD y el JRC (2008), basadas en la implementación de una serie de etapas de forma sucesiva.

La primera de las etapas antes referidas es la "normalización de datos". Este paso es una necesidad previa a cualquier agregación de indicadores, dado que éstos están cuantificados normalmente en diferentes unidades. En nuestro caso, de las diferentes técnicas de normalización existentes, se propone el empleo de la normalización a través de la distancia a una referencia, considerando los valores que se alcanzarían en el caso de que no existiese brecha de género alguna según la siguiente expresión:

$$
I_{k}^{*}=\frac{I_{k}}{I_{k_{-} \text {igual }}}
$$

donde $I_{k}^{*}$ es el valor normalizado del indicador $k, I_{k}$ es el valor de dicho indicador sin normalizar e $I_{\text {kigual }}$ es el valor del mismo indicador en el caso de que se verificase igualdad efectiva entre géneros. De la expresión [1] se deduce cómo valores de $I_{k}^{*}$ inferiores (superiores) a la unidad indican un desequilibrio de género desfavorable (favorable) para las mujeres.

Las siguientes etapas son la "asignación de pesos y la agregación de los indicadores". Ambas etapas son las de mayor importancia dentro del proceso de construcción de indicadores sintéticos, por lo que serán tratadas en detalle en los dos subapartados siguientes.

La última etapa es el "análisis de la relación de los índices con los datos originales", al objeto de identificar los indicadores que presentan una mayor influencia sobre los índices construidos, tarea que será abordada en el apartado de resultados.

La ponderación de los indicadores de base

La etapa de ponderación o asignación de pesos permite distinguir la importancia relativa de los distintos indicadores de base considerados. Para ello, como se expone en OECDJRC (2008), existen múltiples métodos alternativos. Por un lado, las técnicas "endógenas" permiten obtener los pesos de los indicadores de base mediante procedimientos estadísticos, sin que sea necesario incorporar ningún juicio de valor (análisis de componentes principales, análisis de la envolvente de datos y análisis de regresión). Por su parte, las técnicas "exógenas" pretenden asignar pesos diferenciados a los indicadores 
en función de la opinión de expertos o decisores externos (proceso analítico jerárquico, asignación directa de puntos, ponderación de trade-off, el método SMART, etc.).

Para este ejercicio se ha optado por el segundo tipo de técnicas, dado el carácter normativo del concepto de igualdad de géneros. Efectivamente, sólo a través de los juicios de valor expresados por un grupo de personas cualificadas en este sentido puede introducirse en el análisis la importancia relativa de las diferentes áreas temáticas que condicionan la existencia de disparidades entre géneros, así como la importancia diferencial entre los diferentes indicadores de base propuestos para la cuantificación de tales ámbitos.

Teniendo en cuenta la diversidad de técnicas de ponderación "exógenas", se ha seleccionado el proceso analítico jerárquico (Analytic Hierarchy Process en la terminología anglosajona, abreviadamente AHP). Tal decisión no ha sido aleatoria, sino que se ha optado por la técnica que ha resultado más adecuada teniendo en cuenta la estructura jerárquica de los indicadores considerados, así como la necesidad de aplicar un procedimiento realmente operativo a la hora de realizar las entrevistas a un panel de expertos de origen y formación dispar.

La metodología AHP fue creada por Saaty (1980) como técnica estructurada pero flexible para la toma de decisiones en contexto multicriterio. Se basa en la formalización de problemas de decisión complejos empleando una estructura jerárquica a través de una jerarquía que al menos tenga 3 niveles: meta final del problema en lo más alto, los criterios de decisión en un nivel intermedio y las alternativas en la base de la misma. Cuando los criterios son abstractos o complejos, este nivel intermedio de la jerarquía puede a su vez descomponerse en diferentes niveles de subcriterios ordenados secuencialmente. Para el caso de estudio, la jerarquía aplicable se puede plantear a través de 4 niveles: meta final del problema, criterios (objetivos de la igualdad de géneros), subcriterios (indicadores de base) y alternativas (ISIG para cada unidad de análisis considerada), tal y como se expone en la Tabla 3.

Dentro de esta estructura jerárquica, la importancia relativa o ponderaciones entre criterios o subcriterios $\left(w_{i}\right)$ se obtienen sobre la base de comparaciones por pares, determinando la intensidad de preferencia entre cada par de opciones en una escala que va de 1 a 9 . De esta forma, para determinar la importancia relativa de cada uno de los indicadores propuestos, los entrevistados (expertos en temas de igualdad de géneros) han realizado dos tipos de comparaciones: (a) comparaciones por pares de los subcriterios (indicadores) incluidos en cada criterio (objetivo 0 área temática de la igualdad de género), y (b) comparaciones por pares entre criterios (objetivos o área temática). De esta forma, para cada experto entrevistado $(k)$, se han generado 8 matrices de Saaty ${ }^{5}$

\footnotetext{
${ }^{5}$ En total, cada experto participante ha realizado 8 ejercicios de AHP. En primer lugar se han realizado las comparaciones por pares correspondientes a cada una de las 7 áreas temáticas consideradas para analizar la igualdad de género, calculándose así 7 matrices de Saaty y las ponderaciones oportunas de los indicadores. De manera adicional, cada experto ha realizado igualmente las comparaciones por pares para evaluar
} 
con la siguiente estructura:

$$
A_{k}=\left[\begin{array}{cccc}
a_{11_{-} k} & a_{12_{-} k} & \ldots & a_{1 n_{-} k} \\
a_{21_{-} k} & a_{22_{-} k} & \ldots & a_{2 n_{-} k} \\
\ldots & \ldots & a_{i j_{-} k} & \ldots \\
a_{n 1_{-} k} & a_{n 2_{-} k} & \ldots & a_{n n_{-} k}
\end{array}\right]
$$

donde $a_{i j \_}$representa el valor de comparación entre el (sub)criterio $i$ y el (sub)criterio $j$ del experto $k$. Los valores otorgados a las comparaciones por pares representan en realidad ratios entre las ponderaciones concedidas a los correspondientes atributos por un decisor perfectamente racional: $a_{i, j}=w_{i \_} / w_{j \_k}$ para todo $i$ y $j$.

En caso de perfecta consistencia del experto, las $n$ ponderaciones o pesos $\left(w_{i, k}\right)$ de cada (sub)criterio podrían determinarse a partir de los $n(n-1) / 2$ valores de $a_{i j k}$ que éste ha declarado. Sin embargo, la perfecta consistencia raramente se da en la realidad cuando la subjetividad entra en juego. Por ello, para las matrices de Saaty $\left(A_{k}=a_{i j k}\right)$ que presenten cierto grado de inconsistencia, se han propuesto diferentes técnicas destinadas a estimar el vector de pesos que mejor se adapte al vector real de ponderaciones del entrevistado. En este sentido, en este trabajo se ha optado por el método de operativa más simple para la estimación de tales prioridades, como es el de la media geométrica (Aguarón y Moreno-Jiménez 2000). Así, el cálculo de los pesos particulares que cada encuestado asigna a los diferentes atributos se realiza aplicando la siguiente expresión algebraica:

$$
w_{i_{-} k}=\sqrt[n]{\prod_{i=1}^{i=n} a_{i j_{-} k}} \quad \forall \mathrm{i}, \mathrm{j} \in \mathrm{n}
$$

En principio esta técnica del AHP se pensó para decisores individuales, pero pronto se extendió como técnica válida para la decisión de grupos (Easley et al. 2000). Este caso es el que se presenta en este trabajo, en la medida que la ponderación de indicadores debe hacerla un panel de expertos en materia de igualdad de géneros. Así pues, se ha procedido siguiendo las tres fases siguientes: (a) selección del panel de expertos, (b) obtención de las comparaciones por pares y las matrices de Saaty individuales de dichos expertos $\left(A_{k}=a_{i j k}\right)$, así como la estimación de las correspondientes ponderaciones $\left(w_{i \_k}\right)$, y (c) realización de la oportuna síntesis de ponderaciones para el cálculo de los pesos a nivel agregado $\left(w_{i}\right)$.

la importancia relativa de cada una de las áreas temáticas consideras, procedimiento a través del cual se ha generado una nueva matriz de Saaty, de la cual se han derivado igualmente los pesos correspondientes. 
Con este propósito, se ha contado con la colaboración del panel de expertos ya mencionado anteriormente. Estos expertos fueron entrevistados durante el mes de marzo de 2009 al objeto de completar el cuestionario donde se recogían las comparaciones por pares de las 8 matrices de Saaty planteadas.

Una vez obtenidos los valores de las diferentes comparaciones $\left(a_{i j, k}\right)$, se pudieron estimar las correspondientes ponderaciones individuales $\left(w_{i_{k} k}\right)$. Posteriormente se ha realizado la síntesis de la opinión del panel de expertos siguiendo el procedimiento propuesto por Forman y Peniwati (1998), que para decisiones de grupo en el ámbito social sugieren la aplicación del método de agregación de ponderaciones individuales $\left(w_{i \_k}\right)$ estimado a través de la media geométrica:

$$
w_{i}=\sqrt[m]{\prod_{k=1}^{k=m} w_{i_{-} k}}
$$

Finalmente debe comentarse que la estructura jerárquica del AHP motiva que los pesos obtenidos en cada nivel sumen siempre la unidad (por ejemplo véase en la Tabla 3 cómo $w_{P F}+w_{E F}+w_{E R}+w_{P P S}+w_{U T}+w_{C V}+w_{O I O}=1, w_{P F 1}+w_{P F 2}+w_{P F 3}+w_{P F 4}=1$, etc.). Por ello, para poder comparar posteriormente la importancia relativa de los diferentes indicadores propuestos, se hace necesario obtener los correspondientes pesos normalizados $\left(w_{i}^{*}\right)$, tal y como muestra la Tabla 3 . Estos pesos normalizados resultan de multiplicar los pesos de cada subcriterio (indicador en nuestro caso) por la ponderación del criterio jerárquicamente superior (objetivo en nuestro caso); por ejemplo, $w_{P F I}^{*}=w_{P F} \times w_{P F 1}$, $w_{P P S 3}^{*}=w_{P P S} x w_{P P S 3}$, etc. De esta forma los pesos de todos los indicadores debidamente normalizados suman igualmente la unidad, siendo cada $w_{i}^{*}$ un indicador de la importancia del indicador $i$ sobre el conjunto total de indicadores considerados.

\section{La agregación de indicadores}

El procedimiento de agregación resulta controvertido, en la medida en que existen múltiples procedimientos válidos para ello (OECD-JRC 2008), pero que proporcionan resultados dispares. Por este motivo, los estudios empíricos con indicadores sintéticos realizados hasta la fecha han sido criticados por la elección "arbitraria" del procedimiento de agregación. La elección de una forma funcional para la agregación de indicadores no resulta trivial, pues ésta condiciona el tipo de compensación o "tasa marginal de sustitución" entre indicadores. De forma resumida, puede afirmarse que las funciones lineales aditivas asumen implícitamente una compensación total entre indicadores, mientras que las funciones multiplicativas o geométricas admiten una compensación parcial. Para este trabajo se ha considerado conveniente aplicar ambos tipos de formas funcionales, al objeto de poder discutir a partir de los resultados obtenidos las bondades/inconvenientes de uno y otro enfoque para este tipo de estudios. De manera más concreta, las formas funcionales de agregación seleccionadas han sido: 
1. La "suma ponderada de indicadores" (métodos aditivos que asumen compensación total entre indicadores):

$$
I S I G a=\sum_{i=1}^{i=n} w_{i}^{*} I_{i}^{*}
$$

donde ISIGa es el Indicador Sintético de Igualdad de Género de tipo aditivo, $w_{i}^{*}$ es el peso normalizado asociado al indicador $i$, e $l_{i}^{*}$ es el valor normalizado del indicador $i$.

2. El "producto de indicadores ponderados" (métodos de agregación multiplicativos o geométricos que asumen compensación parcial):

$$
I S I G m=\prod_{i=1}^{i=n} I_{i}^{* w_{i}^{*}}
$$

donde ISIGm es el Indicador Sintético de Igualdad de Género de tipo multiplicativo.

\section{Aplicación EmpíRICA}

\section{El conocimiento experto: la ponderación de los indicadores de base}

Con respecto a la obtención de las ponderaciones de los diferentes indicadores, la primera circunstancia a comentar es que para cada experto y conjunto de indicadores considerados (11 expertos $\times 7$ ámbitos temáticos) se han calculado los correspondientes Ratios de Consistencia (CR), como mecanismo de comprobación de que las inconsistencias mostradas por tales expertos en las ponderaciones son aceptables. Así, siguiendo el criterio de Saaty (1980), se ha comprobado que los valores de los diferentes CR han resultado en todos los casos menores del $20 \%$, límite que puede considerarse adecuado para validar los pesos resultantes. Verificada así la validez de los pesos individuales, se ha procedido posteriormente a la pertinente agregación (expresión [4] del apartado anterior).

El resultado final de estos pesos grupales puede observarse en la Tabla 3, donde aparecen calculadas tanto las ponderaciones dadas a cada área temática concreta $\left(W_{i}\right)$, como los pesos asignados a cada indicador con respecto a los demás indicadores de su correspondiente área $\left(w_{i}\right)$ y también con respecto al total de indicadores propuestos en el conjunto de ámbitos socioeconómicos considerados $\left(w_{i}^{*}\right)$. Estos últimos datos $\left(w_{i}^{*}\right)$ serán los que finalmente se utilicen para agregar de manera ponderada cada indicador y poder así construir el ISIG. 
Del análisis de las ponderaciones obtenidas cabe reseñar que el objetivo 0 área temática considerada más importante para el conjunto de expertos entrevistados ha sido la relativa a "Empleo y Renta", con un peso relativo del $24,7 \%$, indicativo del común parecer de que disminuyendo las desigualdades laborales y económicas de hombres y mujeres, puede también reducirse la brecha intergéneros en los demás aspectos. Además, dentro de dicho ámbito, las variables más relevantes han resultado la tasa de paro y la tasa de actividad, revelando que lo realmente importante es acceder a un puesto de trabajo y, posteriormente, conseguir mejores condiciones de empleo.

A continuación, y por orden decreciente de relevancia, se encuentran los objetivos de "Educación y Formación" (18,6\%), "Población y Familia" (15,3\%) y "Calidad de Vida" $(14,7 \%)$, conformando todos ellos las principales condiciones para que las mujeres puedan gozar de un adecuado bienestar personal en las zonas rurales: mayor acceso al mercado laboral, elevado nivel educativo y formativo (preferentemente universitario), mayor proporción de mujeres, principalmente jóvenes y mejor dotación de servicios e infraestructuras.

Los tres objetivos restantes comparten ponderaciones muy parejas: "Participación Política y Social" (9,7\%), "Opiniones sobre la lgualdad de Oportunidades" (8,8\%) y "Usos del Tiempo" (8,1\%). Parece percibirse, por lo tanto, que se trata de objetivos 0 áreas instrumentales necesarias para alcanzar la igualdad, dada su apreciable ponderación, pero que ostentan una menor importancia relativa en relación con los objetivos más apreciados. Asimismo, se ponderan en mayor grado todas aquellas variables que tienen que ver con el mercado laboral: opiniones a favor de una mayor responsabilidad de las mujeres en el trabajo y el mayor espacio de tiempo dedicado al trabajo remunerado. Finalmente, se destaca también la opinión mayoritaria en torno a la necesidad de que las mujeres ocupen cargos políticos en una mayor proporción, con un $42,1 \%$ de importancia relativa dentro de su correspondiente área temática. 


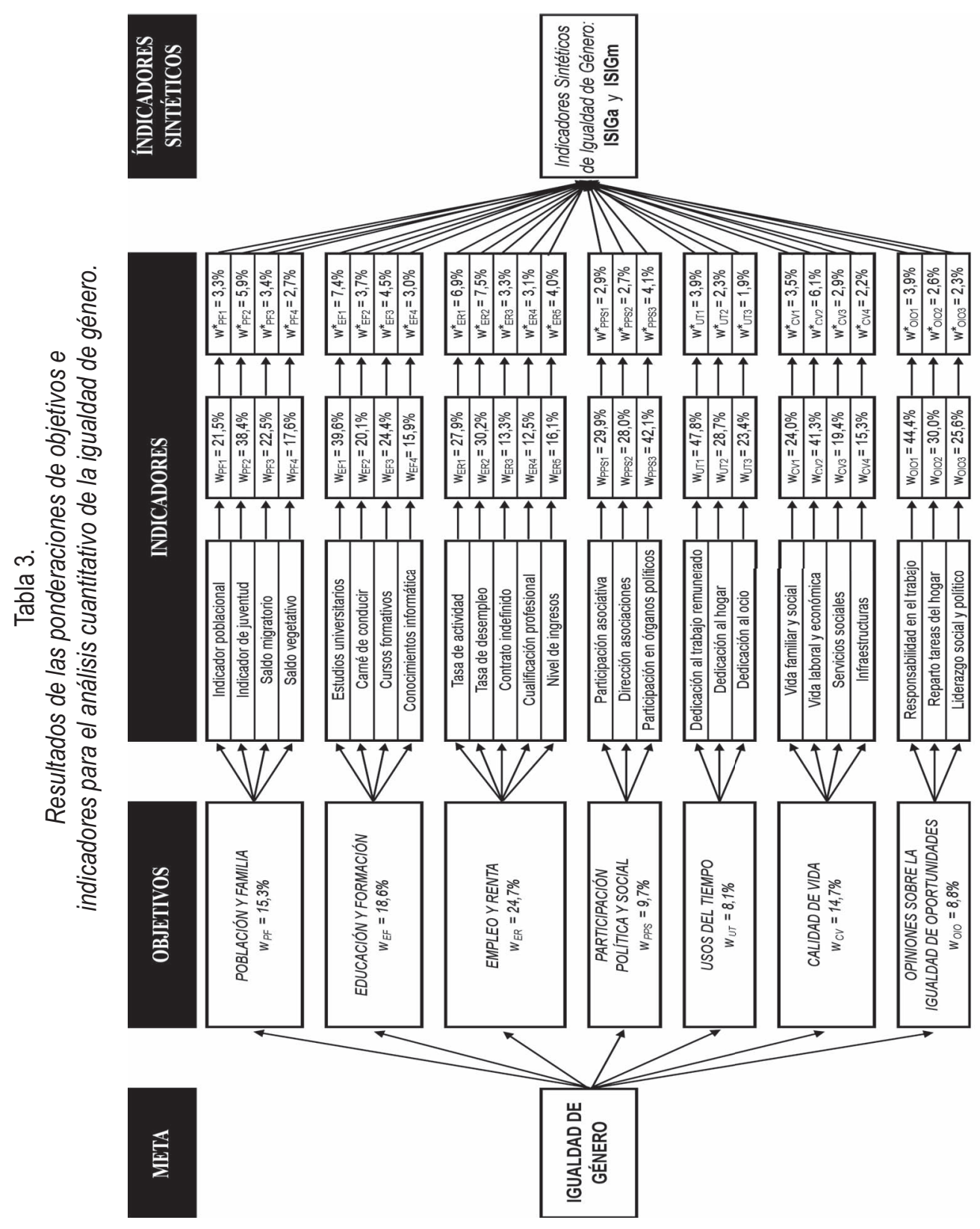




\section{Cálculo de los indicadores de base}

La construcción de indicadores sintéticos requiere el cálculo previo de los indicadores simples que aparecen en la Tabla $4\left(\mathrm{I}_{\mathrm{k}}{ }^{*}\right)$, agrupados en función de sus respectivas áreas temáticas y por hábitats. Así, se han calculado los porcentajes o valores de las variables que intervienen en el cálculo de los indicadores, tanto para mujeres (M) como para varones (V). En una columna posterior se han obtenido los valores de los correspondientes indicadores de igualdad, como el cociente entre ambos porcentajes o valores (porcentaje mujeres/porcentaje varones). Así, valores de $I_{k}{ }^{*}$ inferiores al $100 \%$ implican una desigualdad a favor del colectivo masculino y a la inversa. Para el caso de variables del tipo "cuanto menor mejor", como por ejemplo la tasa de paro, el indicador se ha calculado como la razón entre el porcentaje o valor de los varones y el de las mujeres. Asimismo, para las variables pertenecientes al área de "Opiniones sobre la Igualdad" se ha establecido la hipótesis de que se alcanza un mayor nivel de igualdad si ambos sexos, en conjunto, opinan que hay que desterrar los estereotipos prefijados acerca de las mujeres. Por ello, el indicador de desigualdad en este caso se ha calculado como la distancia porcentual entre las opiniones medias vertidas por los hombres y las mujeres acerca de este tema y el objetivo ideal (valor del indicador del 100\%). 


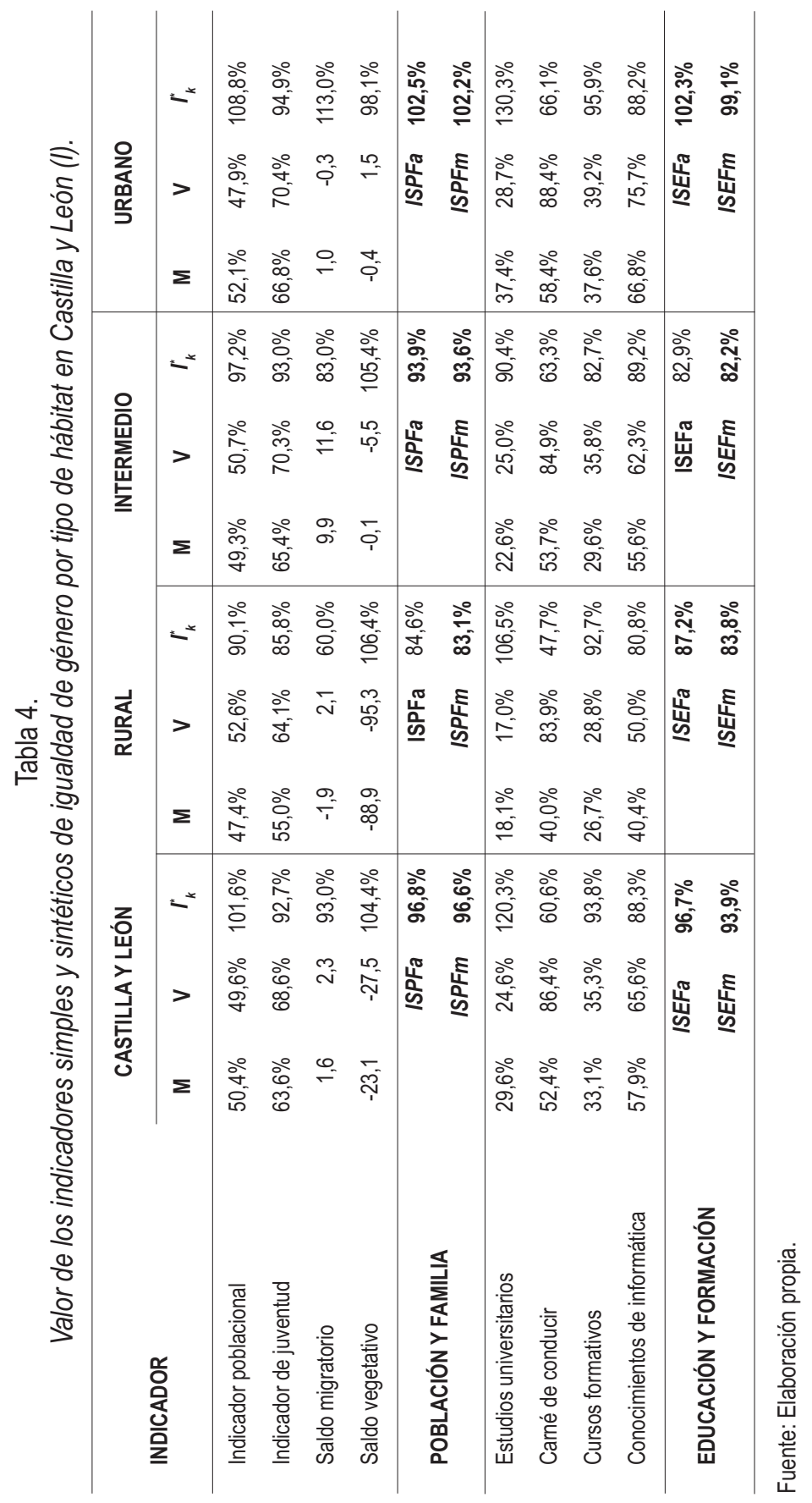




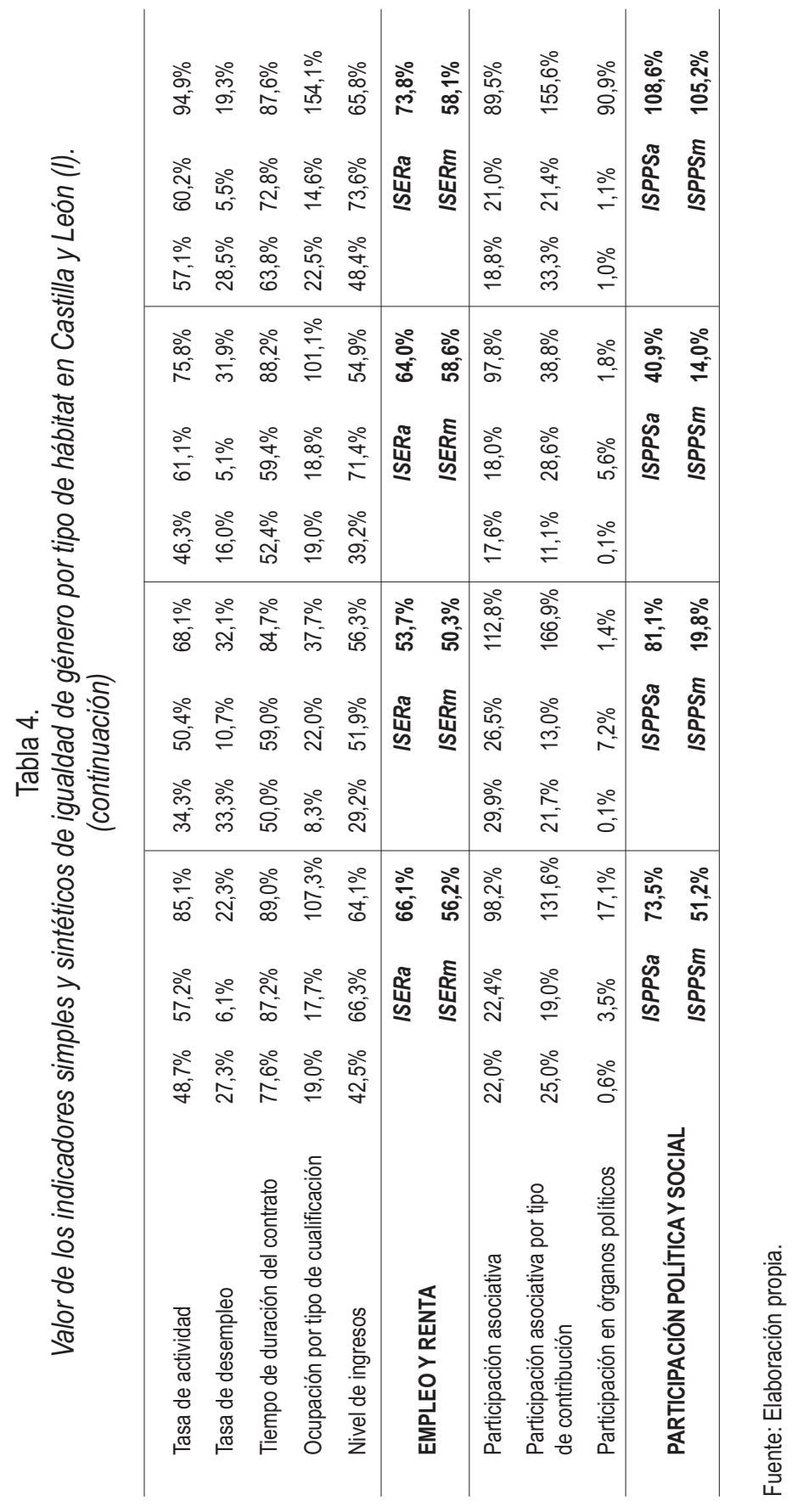




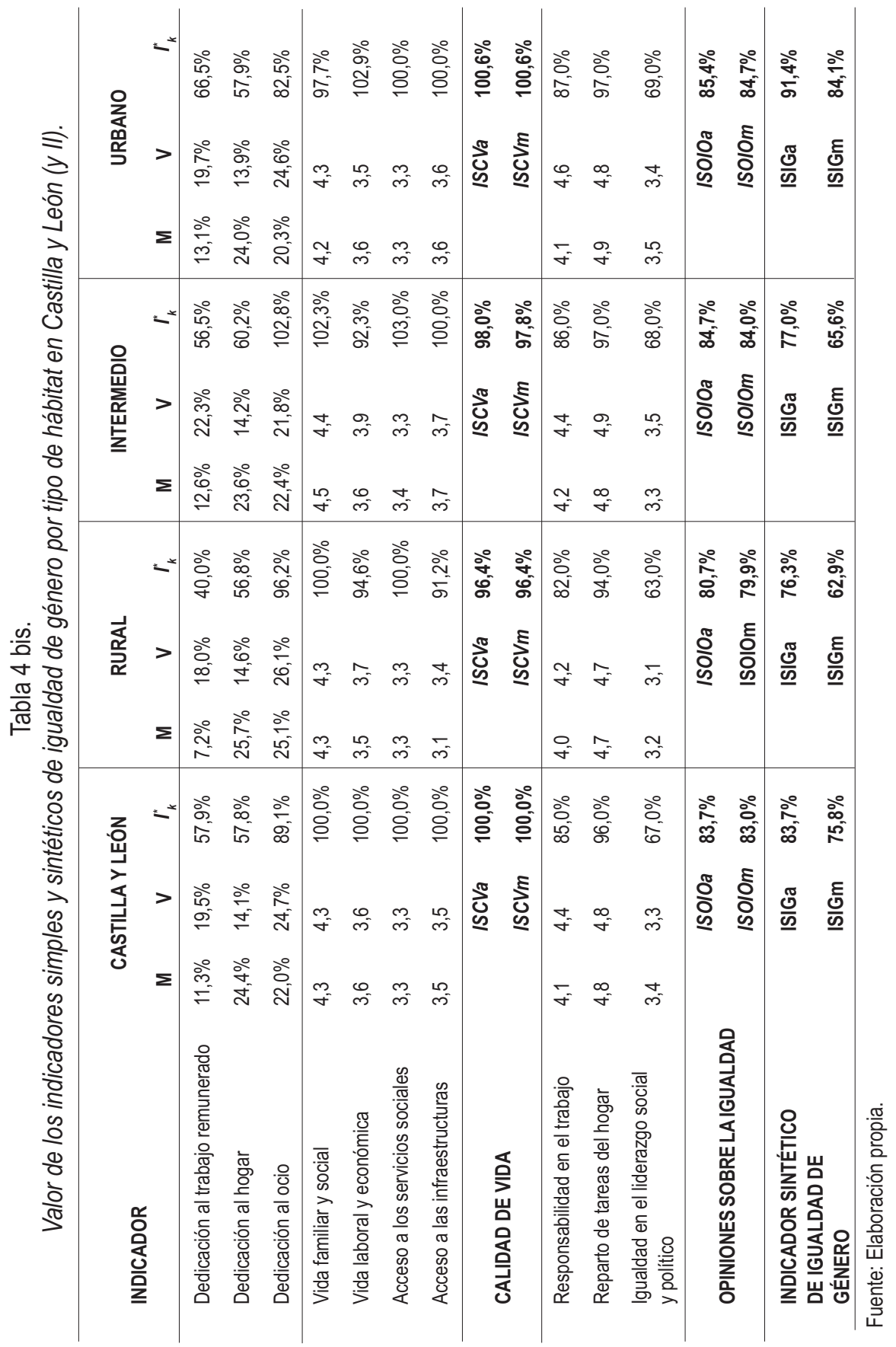




\section{Resultados de los indicadores sintéticos}

\section{Resultados por tipo de hábitats}

Los valores de los indicadores sintéticos de igualdad de género finalmente obtenidos, tanto para el conjunto regional de Castilla y León como para cada unos de los hábitats considerados, pueden observarse junto a los indicadores de base en la Tabla 4 (dos últimas filas). Así, tal y como se especificó en el apartado metodológico, se ha calculado el indicador de forma aditiva (ISIGa) y también de forma multiplicativa (ISIGm). Adicionalmente, en dicha tabla se han recogido los indicadores sintéticos parciales de cada una de las áreas temáticas referenciadas.

No obstante, antes de comentar los resultados obtenidos, debe señalarse que el error muestral de los indicadores de partida calculados para cada tipo de hábitat se incrementa sensiblemente con respecto a los apuntados en la Tabla 2 (ficha técnica de la encuesta), alcanzando casi el $10 \%$. Por este motivo, los resultados referidos a continuación deben considerarse con la debida cautela ${ }^{6}$. De cualquier forma, esta circunstancia en ningún caso invalida el enfoque metodológico propuesto, como principal aportación de la presente investigación.

Del análisis de los resultados alcanzados puede concluirse que, tal y como cabía esperar a tenor de los distintos estudios existentes sobre el tema, así como del análisis previo de los indicadores de base, existe una severa desigualdad socioeconómica entre mujeres y varones en el conjunto del territorio de Castilla y León, independientemente del grado de ruralidad concurrente (valor de los ISIG inferiores al 100\%). Así, para el conjunto regional el valor del ISIGa asciende a 83,7\%, mientras que el ISIGm alcanza el 75,8\%.

En cualquier caso, se puede establecer una jerarquía de la brecha de género según el tipo de hábitat, la cual estaría encabezada por las zonas rurales, en la que se originan los diferenciales más acusados (ISIGa =76,3\%; ISIGm =62,9\%), seguida del área intermedia, en ambos casos con valores de los ISIG por debajo de la media regional. EI espacio que mejor se comporta con respecto a la igualdad entre géneros es el medio urbano, el único territorio con valores de los ISIG superiores al conjunto de la región (ISIGa = 91,4\%; ISIGm = 84,1\%).

\footnotetext{
${ }^{6}$ La utilización de únicamente dos estratos de hábitat (rural y urbano) en vez de tres mejoraría los errores de significación de los indicadores sintéticos obtenidos. Sin embargo, tal opción enmascararía las grandes diferencias en lo que a igualdad de género se refiere entre los pequeños municipios rurales y los municipios intermedios o cabeceras de comarca. Esta circunstancia se magnifica en Castilla y León, habida cuenta de la gran cantidad de municipios pequeños que hay y de la mayor población rural que allí existe en relación a otras Comunidades Autónomas. Por este motivo para esta investigación, donde la propuesta metodológica prima sobre la precisión estadística, se ha considerado más conveniente hacer la estratificación para la consideración diferencial de los tres tipos de hábitat comentados. En cualquier caso, la estratificación por hábitats, edades o cualquier otra variable sociodemográfica considerada debería hacerse teniendo en cuenta las características propias de la sociedad analizada.
} 
Tal y como se ha apuntado, dicha jerarquía se mantiene al observar tanto los valores del ISIGa como del ISIGm. En este sentido, resulta interesante comentar que este último índice determina la persistencia de mayores disparidades entre los sexos (valores de ISIGm menores que ISIGa) en todas las áreas temáticas y en todos los espacios geográficos. Este hecho se debe a la capacidad de compensación implícita entre los distintos indicadores que componen el ISIGa, lo que hace que la desigualdad que se registra en alguna de las variables se compense con la menor desigualdad de otras y que, finalmente, la asimetría global no sea muy acentuada. Así, la diferencia entre este indicador de tipo aditivo y los valores del ISIGm lo que refleja realmente es el desequilibrio existente entre los indicadores simples considerados.

Atendiendo exclusivamente a los datos del medio rural castellano y leonés, el primer resultado a tener en cuenta es la existencia de una acentuada desigualdad entre hombres y mujeres en todas las esferas socioeconómicas tenidas en cuenta en el estudio. En este sentido, las mayores disparidades se observan en los ámbitos de "Empleo y Renta", "Usos del Tiempo" y "Participación Política y Social". En este último caso, destaca el amplio contraste entre los valores del ISIGa y del ISIGm, debido a la compensación que se da en el primero entre las variables de participación asociativa (acusada desigualdad a favor de las mujeres) y de participación política (severa desigualdad en contra de las mujeres). Estos resultados están en sintonía con los problemas y necesidades reivindicadas tradicionalmente por los colectivos de mujeres rurales (Langreo y Benito 2005; Rico 2006), que reclaman a los poderes públicos medidas para alcanzar una igualdad efectiva en el acceso al empleo, la posibilidad de compatibilizar la vida laboral con la vida familiar y la opción de acceder en mayor grado a los puestos de decisión de las organizaciones políticas y sociales. Por su parte, donde se originan las discrepancias intergénero más reducidas es en el área de "Calidad de Vida". No obstante, en este sentido conviene comentar que al tratarse de datos cualitativos medidos en forma de satisfacción personal percibida acerca de varios aspectos vitales, lo que se pone de manifiesto es que, en general, los hombres y las mujeres valoran de manera muy similar las condiciones necesarias para alcanzar un mayor bienestar en las zonas rurales. Finalmente, cabe apuntar que en las sociedades rurales de la región aún se mantiene con cierta intensidad una mentalidad tendente a perpetuar los estereotipos diferenciadores entre hombres y mujeres, ya que los resultados de los indicadores sintéticos obtenidos en dicho área manifiestan la existencia de una opinión bastante generalizada acerca de las menores posibilidades que tiene el colectivo femenino para desempeñar puestos de mayor responsabilidad laboral, así como cargos de liderazgo político.

Por su parte, las zonas denominadas intermedias siguen unas pautas de comportamiento similares a las descritas para la zona rural, si bien, por lo general, el grado de asimetría entre géneros es más reducido. Nuevamente, las brechas de género más acusadas se reproducen en las esferas de "Empleo y Renta", "Usos del Tiempo" y "Participación Política y Social", con diferencias considerables entre los índices aditivo y multiplicativo en ese último área, también debido a la desequilibrada participación social de las mujeres con respecto a su desempeño político. Por su parte, es preciso destacar 
que las mínimas diferencias detectadas entre los ISIG del hábitat intermedio y los ISIG de la zona rural se relacionan exclusivamente con el mayor movimiento asociativo femenino de los municipios más pequeños, a tenor de los datos recabados en la encuesta realizada para este trabajo. Este hecho puede explicarse debido a que, al tratarse de municipios muy pequeños, la mayor parte de las mujeres (muchas de ellas inactivas laboralmente) pertenecen a las pocas asociaciones existentes en su pueblo, sean de la naturaleza que sean, con lo que estadísticamente la participación asociativa femenina es muy elevada. Sin embargo, los demás resultados muestran las grandes discrepancias que existen tradicionalmente entre unos y otros territorios y, especialmente, en ámbitos tan relevantes como son los de "Población y Familia" y "Empleo y Renta".

Por último cabe comentar cómo es en las grandes urbes donde se registran menores niveles de desigualdad. De hecho, en estos ámbitos se observan valores de los ISIG superiores al $100 \%$ en alguna de las dimensiones consideradas. Así ocurre en el área de "Población y Familia" (por la mayor proporción de mujeres y sus saldos migratorios positivos), "Educación y Formación" (por la mayor cantidad de mujeres universitarias) e incluso "Participación Política y Social" (por la mayor proporción de mujeres que forman parte de la directiva de las asociaciones). Sin embargo, persisten brechas de género significativas en el ámbito considerado prioritario por el conjunto de expertos consultados, como es el de "Empleo y Renta", así como en los bloques de "Usos del Tiempo" y de "Opiniones sobre lgualdad".

En conclusión, los ISIG obtenidos confirman el mayor grado de desigualdad entre varones y mujeres a medida que la ruralidad del territorio es mayor, y esta circunstancia se reproduce en todas las esferas de la vida económica y social. A este respecto, de la observación de los diferentes indicadores calculados de forma aditiva podría admitirse la existencia de menores disparidades entre géneros en algunas de las parcelas socioeconómicas analizadas, dada la compensación que se produce entre las variables que lo integran. Sin embargo, los índices multiplicativos, donde no se producen esas compensaciones, no dejan lugar a dudas de la magnitud del problema.

Consecuentemente y desde un punto de vista metodológico, cabe señalar cómo la formulación matemática de las formas de agregación alternativas utilizadas (aditiva y multiplicativa, según las expresiones [5] y [6]), motiva que en todos los casos los índices agregados de forma aditiva tomen valores superiores a los agregados de forma multiplicativa. Además, también motivado por su forma de cálculo, la diferencia entre uno y otro valor se ve incrementada en la medida que aumenta la variabilidad interna de los indicadores a agregar (a mayor heterogeneidad de los indicadores de base, el valor de la agregación multiplicativa se hace menor). Estas circunstancias son, en definitiva, el reflejo del carácter plenamente compensatorio de la agregación aditiva (los valores más bajos de los indicadores a agregar se "corrigen" totalmente con los valores mayores), en comparación con la compensación parcial de los índices multiplicativos (los valores menores de los indicadores sólo pueden "corregirse" en parte por los valores más elevados).

Así pues, las preferencias en relación a uno u otro tipo de indicadores sintéticos dependerá de la conceptualización que se haga de la igualdad de género, bien como 
una agregación de dimensiones y atributos conmensurables (compensables entre sí a través de tasas de intercambio determinadas), o bien como un concepto complejo de dimensiones y atributos inconmensurables (no se acepta la compensación entre los diferentes indicadores). Ambas acepciones de la igualdad de género pueden considerarse válidas, sin que quepa optar de forma objetiva por una de ellas. Por este motivo, parece conveniente que este tipo de análisis se realice considerando conjuntamente ambos tipos de indicadores compuestos, teniendo en cuenta la significación diferencial de cada uno de ellos, tal y como se ha señalado anteriormente.

\section{Resultados por tramos de edad}

A continuación, y con el fin de efectuar un análisis dinámico acerca de la evolución de las desigualdades entre mujeres y hombres y los posibles cambios sociales acontecidos en lo que se refiere a la relación entre géneros, se han efectuado los distintos cálculos para tres grupos distintos de edad: "población joven" (entre 16 y 29 años), "población madura" (entre 30 y 64 años) y "población mayor" (más de 64 años). Los resultados así obtenidos permitirán determinar si las brechas de género identificadas para la población en su conjunto persisten en todas las generaciones o dicho fenómeno tiende a remitir a medida que la modernización de las distintas sociedades se va generalizando (Tabla 5). Nuevamente debe destacarse que el error muestral de los indicadores de partida calculados para cada cohorte de edad se incrementa considerablemente con respecto a los apuntados en la ficha técnica de la encuesta. Por este motivo, los resultados alcanzados deben considerarse igualmente con cautela.

En consecuencia, puede afirmarse que las disparidades por razón de género son mucho más reducidas para la población joven en comparación con los demás estratos de edad, para todos los ámbitos socioeconómicos y en todos los hábitats analizados. Los valores del ISIGa para este grupo poblacional se aproximan bastante al $100 \%$, lo que refleja un escaso grado de desigualdad global de género entre los jóvenes. Así, si bien aún la población femenina no se ha equiparado completamente con la masculina en ninguna de las dimensiones consideradas, las brechas que les separa son más reducidas con respecto a generaciones anteriores.

También en el caso de este grupo de individuos los desajustes entre hombres y mujeres se manifiestan con mayor intensidad en las zonas rurales, al igual que en las áreas intermedias, siendo en las grandes ciudades donde los contrastes son más reducidos. No obstante, este resultado parece confirmar el cambio de rumbo que están tomando las nuevas generaciones de mujeres rurales y sus deseos de permanecer en el territorio, pero de manera muy diferente a como lo hicieron sus madres. Las mujeres rurales de hoy en día desean incorporarse al mercado de trabajo reglado, participar en la vida social y política de sus pueblos y, consiguientemente, actuar de la manera más pareja posible a los hombres de su misma edad (Díaz Méndez 2006). No obstante, aún queda un significativo camino por recorrer para que se consiga la igualdad efectiva en todas y cada una de las esferas de la vida de estas mujeres. 


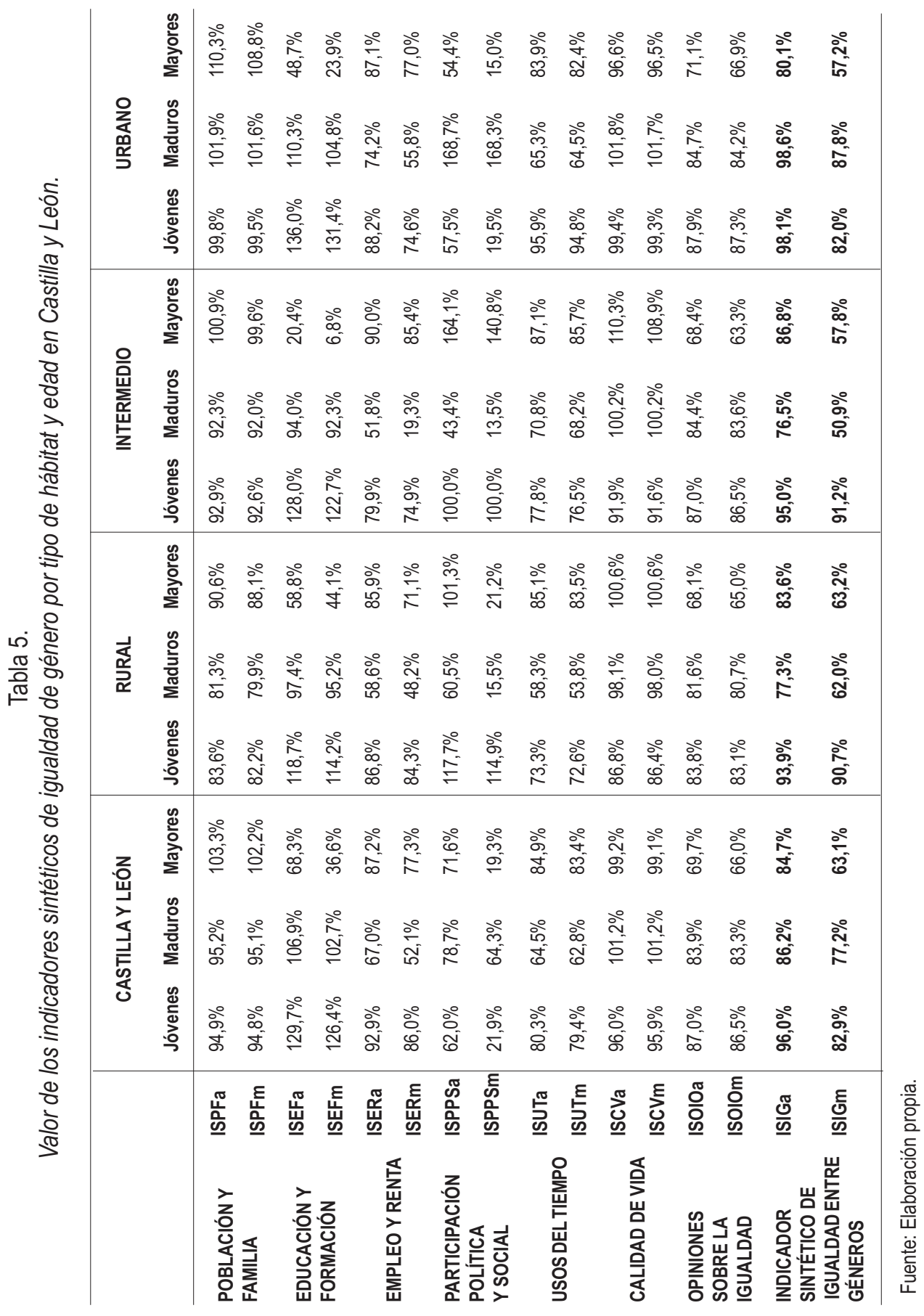


Para la población madura, los valores de los índices se reducen de forma importante, sobre todo en los territorios rurales e intermedios, debido a la intensa disparidad intergénero registrada en los ámbitos de "Empleo y Renta", "Participación Política y Social" y "Usos del Tiempo". Se trata, en este caso, del colectivo de mujeres que más ha sufrido la crisis de las zonas rurales y que, sin optar por emigrar a las ciudades, decidieron quedarse en sus municipios de origen pero en unas condiciones que revelan su invisibilidad laboral, económica y social. En el caso de la población mayor, la jerarquía territorial difiere de la observada para los demás estratos de edad. Así, en esta ocasión los menores valores de los ISIG se encuentran en la zona urbana, siendo superior en los espacios rurales e intermedios. Esta circunstancia responde principalmente a la escasa participación política y social de las mujeres en las urbes en comparación con los varones. No obstante, al observar únicamente el $I S I G m$, las diferencias entre unos y otros hábitats son mucho más reducidas, lo que no permite realizar afirmaciones demasiado categóricas al respecto.

\section{CONCLUSIONES}

La actual desigualdad económica y social debida al género que se padece actualmente en todos los países en general, y en las zonas rurales en particular, requiere una especial necesidad de intervención pública, que debe guiarse en función de información técnica procedente de estudios realizados sobre la base de indicadores específicos en este sentido. A este respecto, una primera conclusión derivada del trabajo es la constatación de la insuficiente información pública disponible, convenientemente desagregada por sexo y tipo de hábitat, para la realización de este tipo de análisis de carácter multidimensional. Estas carencias son especialmente relevantes en los ámbitos de la formación, el mercado laboral, la calidad de vida o la percepción acerca de la igualdad de oportunidades. El sistema de indicadores propuesto para este análisis, así como el cuestionario ad hoc en el que se basa parte del estudio empírico implementado, pueden constituir una orientación válida sobre las lagunas informativas que deberían ser cubiertas en un futuro próximo por parte de las diferentes administraciones.

Dado que la disparidad de géneros es un problema multidimensional, en el que entran a formar parte numerosas facetas de una misma realidad, se estima oportuno calcular y analizar indicadores sintéticos, al objeto de aglutinar y facilitar la interpretación de la múltiple información de que se dispone. Desde este punto de vista, el indicador sintético de igualdad de género planteado en este trabajo puede constituir una herramienta útil para las administraciones públicas encargadas de aplicar y evaluar medidas tendentes a corregir las disparidades entre hombres y mujeres en distintos ámbitos socioeconómicos y geográficos. La aplicación de este tipo de estudios estadísticos sobre hábitats heterogéneos resulta esencial para determinar los desfases interterritoriales existentes, procurando a los poderes públicos una valiosa información para actuar en consecuencia 
y procurar al conjunto de la población un nivel de vida adecuado y equilibrado entre géneros, independientemente del lugar en el que resida.

Atendiendo al caso concreto objeto de estudio, se ha podido constatar y cuantificar el grado de asimetría de género registrado en el medio rural de Castilla y León, que ha resultado ser significativamente mayor al de otros espacios más poblados. Adicionalmente se ha verificado que esa desigualdad va remitiendo con el paso del tiempo, de manera que la asimetría de género es más exigua para las generaciones de población joven en relación a las generaciones de edad superior. Esta circunstancia parece reflejar el importante cambio generacional que se está produciendo en las zonas rurales, en el sentido de que las mujeres más jóvenes tienen una mayor formación y unos mayores deseos de incorporación al mercado de trabajo y en consecuencia tratan de nivelar su situación económica y social con la de sus congéneres varones. Consecuentemente, se puede afirmar que, aunque lentamente, las distintas políticas de igualdad están alcanzando los objetivos planteados en relación a la progresiva equiparación económica y social entre hombres y mujeres. No obstante, aún quedan importantes obstáculos que superar, en especial aquellos relacionados con el cambio de mentalidad de la sociedad en cuanto a los estereotipos y roles asignados a las mujeres. Hombres y mujeres son iguales y, ante las mismas circunstancias, han de estar en igualdad de condiciones para lograr sus metas.

Por su parte, cabe manifestar que si bien los resultados alcanzados (la peor situación de las mujeres que habitan en los municipios pequeños) es un hecho suficientemente constatado en estudios anteriores, es necesario señalar que lo verdaderamente innovador de esta investigación es la propuesta metodológica de medición de la desigualdad a través del uso de indicadores sintéticos. En este sentido, la coincidencia de los resultados empíricos obtenidos con los reportados en la literatura existente no cabe sino interpretarlos como una validación del enfoque cuantitativo propuesto. Asimismo, se ha demostrado las amplias posibilidades que plantea la construcción y aplicación de este tipo de índices, tal y como ha quedado reflejado en la aplicación empírica realizada. La utilidad de su uso radica en la relativa sencillez para su elaboración, en su capacidad de agrupar y resumir la información, en su posibilidad de aplicación a situaciones reales, en su potencialidad para realizar comparaciones entre diversos tipos de poblaciones y de hábitats y en su capacidad para realizar análisis dinámicos.

Finalmente, cabe apuntar que este trabajo es una primera aproximación a un tema con múltiples posibilidades de desarrollo futuro, en el que se ha querido proponer una metodología válida y eficaz para cuantificar la desigualdad de género en el espacio y en el tiempo. Se abre con ello una vía de investigación en la que se sugiere avanzar, y perfeccionar la construcción de indicadores sintéticos de igualdad de género por medio de otras técnicas estadísticas y la comparación de resultados con los aquí obtenidos. Asimismo, se propone extender el análisis diferenciando a la población según otro tipo de variables, además de la edad, como es su nivel educativo, sus características laborales o su nivel de renta. Finalmente, se propone también calcular los indicadores sintéticos de igualdad de género realizando desagregaciones territoriales desde el punto de 
vista interregional, provincial o comarcal, dependiendo de la cobertura geográfica de la estrategia de desarrollo para la cual quieran ser utilizados.

\section{RefERENCIAS BiBLIOGRÁfICAS}

Agencia Canadiense de Desarrollo Internacional (ACDI). 1996. Guide to gender sensitive indicators. Quebec. Agencia Canadiense de Desarrollo Internacional.

Aguarón, J. y J. M. Moreno-Jiménez. 2000. "Stability Intervals in the Analytic Hierarchy Process." European Journal of Operational Research 125:114-133.

Álvaro, M. 1994. "Marco general para la creación y puesta en marcha de un sistema de indicadores sobre la igualdad de oportunidades de las mujeres." Pp. 19-21, en Propuesta de un sistema de indicadores sociales de igualdad entre géneros, coordinado por M. Álvaro. Madrid: Instituto de la Mujer.

Bericat, E. y E. Sánchez. 2008. Balance de la desigualdad de género en España. Un sistema de indicadores sociales. Sevilla: Centro de Estudios Andaluces.

Boserup, E. 1970. Women's Role in Economic Development. Nueva York: St. Martin's Press.

Calatrava, J. 2002. "Mujer y desarrollo rural en la globalización: de los proyectos asistenciales a la planificación de género." Información Comercial Española 803:73-90.

Camarero, L., coord. 2006. El trabajo desvelado. Trayectorias ocupacionales de las mujeres rurales en España. Madrid: Ministerio de Trabajo y Asuntos Sociales.

Camarero, L. y R. Sampedro. 2008. “¿Por qué se van las mujeres? El continuum de movilidad como hipótesis explicativa de la masculinización rural." Revista Española de Investigaciones Sociológicas 124:73-105.

Cànoves, G.; M. D. García Ramón y M. Solsona. 1989. "Mujeres agricultoras, esposas agricultoras: un trabajo invisible en las explotaciones familiares." Revista de Estudios Agro-Sociales 147:45-69.

Castañeda, I.; M. E. Astraín; V. Martínez y L. Artiles. 1999. "Indicador sintético para medir diferencias de género." Revista Cubana de Salud Pública 25:54-63.

Comisión Económica para América Latina y el Caribe (Cepal). 2006. Guía de asistencia técnica para la producción y el uso de indicadores de género. Santiago de Chile: CEPAL LC/R.2136.

Colectivo loé. 2008. Barómetro social de España. Madrid: Colectivo loé.

Comisión Europea. 2006. Plan de Trabajo para la lgualdad entre las mujeres y los hombres. (20062010). $\operatorname{COM}(2006) 92$ final.

Consejo Económico y Social de Castilla y León (Cescyl). 2004. Las mujeres en el medio rural de Castilla y León. Valladolid: Cescyl. 
Cruz, F. 2006. Género, psicología y desarrollo rural: la construcción de nuevas identidades. Madrid: Ministerio de Agricultura, Pesca y Alimentación.

Dávila, M. 2004. Indicadores de género: guía práctica. Sevilla: Instituto Andaluz de la Mujer.

Díaz Méndez, C. 2006. "Cambios generacionales en las estrategias de inserción sociolaboral de las jóvenes rurales." Revista Española de Estudios Agrosociales y Pesqueros 211:307-337.

Easley, R. F.; J. S. Valacich y M. A. Venkataramanan. 2000. "Capturing Group Preferences in a Multicriteria Decision." European Journal of Operational Research 125:73-83.

Food and Agriculture Organization of the United Nations (FAO). 2001. Gender sensitive indicators: A key tool for gender mainstreaming. Rome: Women in Development Service (SDWW), Women and Population Division.

Forman, E. y K. Peniwati. 1998. "Aggregating Individual Judgments and Priorities with the Analytic Hierarchy Process." European Journal of Operational Research 108:165-169.

Fuente, G. De la. 1987. "Las jóvenes rurales en la encrucijada del cambio (el caso castellano)." Agricultura y Sociedad 42:47-71.

García Bartolomé, J. M. 1990. La mujer agricultora ante el futuro del mundo rural. Tesis Doctoral, Departamento de Estructura Social, Universidad Complutense. Madrid.

García Ferrando, M. 1977. Mujer y sociedad rural. Madrid: Cuadernos para el Diálogo.

García Ramón, M. D. y M. Baylina, comps. 2000. El nuevo papel de las mujeres en el desarrollo rural. Barcelona: Oikos-tau.

García Sanz, B. 1998. La sociedad rural de Castilla y León ante el próximo siglo. Valladolid: Junta de Castilla y León.

García Sanz, B. 2004. La mujer rural ante el reto de la modernización de la sociedad rural. Madrid: Ministerio de Trabajo y Asuntos Sociales.

Junta de Castilla y León. 2007. IV Plan de Igualdad entre mujeres y hombres 2007-2011. Valladolid: Consejería de Familia e Igualdad de Oportunidades.

Jütting, J. P.; C. Morrisson; J. Dayton-Johnson y D. Drechsler. 2006. Measuring Gender (in)equality: Introducting the Gender, Institutions and Development Data Base. París: DEV/DOC(2006)01. OECD.

Langreo, A. e I. Benito. 2005. "La mujer en la agricultura y en el medio rural." Pp 104-128 en Agricultura Familiar en España 2005. Madrid: Fundación de Estudios Rurales.

Instituto de la Mujer. 1995. Carta para la Igualdad de Oportunidades de las Mujeres en el Ámbito Rural. Madrid: Ministerio de Trabajo y Asuntos Sociales.

Instituto de la Mujer. 1999. Mujeres y sociedad rural. Entre la inercia y la ruptura. Madrid: Ministerio de Trabajo y Asuntos Sociales. 
Ministerio de Agricultura, Pesca y Alimentación. 2007. Plan de igualdad entre mujeres y hombres en el medio rural. Madrid: Dirección General de Desarrollo Rural.

Ministerio de Igualdad. 2007. Plan Estratégico de Igualdad de Oportunidades 2008-2011. Madrid.

Organización de las Naciones Unidas (ONU). 1984. Improving Concepts and Methods for Statistics and Indicators on the Situation of Women. Nueva York: Department of International Economic and Social Affairs.

Organización de las Naciones Unidas (ONU). 1995. Informe de la Cuarta Conferencia Mundial sobre la Mujer Beijing Nueva York: A/CONF.177/20/Rev.1. ONU.

Organization for Economic Co-operation and Development \& Joint Research Centre (OECD-JRC). 2008. Handbook on constructing composite indicators. Methodology and user guide. París: OECD.

Programa de Naciones Unidas para el Desarrollo (PNUD). 2007. Informe sobre desarrollo humano 2007-2008. Nueva York: PNUD.

Rico, M. 2006. La contribución económica de la mujer al desarrollo del medio rural de Castilla y León. Un análisis aplicado al turismo rural. Tesis Doctoral, Departamento de Economía Aplicada, Universidad de Valladolid: Valladolid.

Rico, M. y J. A. Gómez-Limón. 2008. "Sociedad y desarrollo rural en Castilla y León: un estudio de opinión pública." Boletín de la Asociación de Geógrafos Españoles 48:199-223.

Saaty, T. L. 1980. The Analytic Hierarchy Process. Nueva York: McGraw-Hill.

Sabaté, A. 1989. Las mujeres en el medio rural. Madrid: Ministerio de Asuntos Sociales.

Sampedro, R. 1996. Género y ruralidad. Las mujeres ante el reto de la desagrarización. Madrid: Ministerio de Trabajo y Asuntos Sociales.

Sancho, J. y D. Reinoso. 2003. "Población y doblamiento rural de España: un primer análisis a la luz del Censo 2001." Serie Geográfica 11:163-176.

Social Watch. 2009. Índice de Equidad de Género 2008. Montevideo: Instituto del Tercer Mundo. Consultado 8 de marzo de 2009 (http:// www.socialwatch.org/es/node/11566).

United Nations Economic Commision for Africa (Uneca). 2004. The African Gender and Economic Index, Addis Ababa: Uneca.

Vera, A. y J. Rivera, J. 1999. Contribución invisible de las mujeres a la economía: el caso específico del mundo rural. Madrid: Instituto de la Mujer.

Vicente-Mazariegos, J. I., coord. 1993. La situación de la mujer en la agricultura. Madrid: Ministerio de Agricultura, Pesca y Alimentación.

World Economic Forum. 2008. The Global Gender Gap Report 2008. Geneva: World Economic Forum. 
MARGARITA RICO GONZÁLEZ es profesora contratada Doctor de la Universidad de Valladolid en el área de Economía Agraria. Su línea investigadora está vinculada al estudio económico y social del medio rural y en especial al análisis del colectivo de mujeres que viven en esas zonas y su contribución al desarrollo económico.

JOSÉ ANTONIO GÓMEZ-LIMÓN RODRÍGUEZ es investigador titular del Instituto de Investigación y Formación Agraria y Pesquera (IFAPA) de la Junta de Andalucía, en el área de Economía y Sociología Agraria. Ha desarrollado su actividad investigadora principalmente en el campo del análisis de políticas agrarias, con especial interés en los aspectos medioambientales (gestión del agua y sostenibilidad) y sociales (desarrollo rural).

\section{RECIBIDO: $27 / 04 / 09$}

ACEPTADO: $17 / 04 / 10$

Publicado on-line: 25/11/10 